\title{
Experimental evaluation of bottleneck link utilization with new-additive increase multiplicative decrease congestion avoidance and control algorithm.
}

\begin{abstract}
Problem statement: As the Internet becomes increasingly heterogeneous, the issue of congestion control becomes ever more important. And the link utilization is one of the important things in term of congestion avoidance and control mechanisms. And we can define the utilization as simply the throughput divided by the access rate. And also all the developments for the congestion control and avoidance algorithms interest about the using of network resources and use the links capacity (utilization). Approach: In this research we continued to study the performances of the New-Additive Increase Multiplicative Decrease (AIMD) algorithm as one of the core protocols for TCP congestion avoidance and control mechanism, we want now to evaluate the effect of using the AIMD algorithm after developing it to find a new approach, as we called it the New-AIMD algorithm to measure the bottleneck link utilization and use the NCTUns simulator to get the results after make the modification of the mechanism. And we will use the Droptail mechanism as Active Queue Management (AQM) in the bottleneck router. Results: After implementation of our new approach with different number of flows, we will measure the bottleneck link utilization and we will get high utilization (more than 94\%)for bottleneck link with using this mechanism and avoid the collisions in the link. Conclusion: Now and after got this results as high utilization for bottleneck link, we know the New-AIMD mechanism work as well under the giving network condition in the experiments.
\end{abstract}

Keyword: Congestion control; TCP; AIMD; Network utilization. 